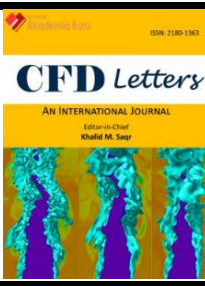

\title{
Computational Fluid Dynamics Study of Wake Recovery for Flow Across Hydrokinetic Turbine at Different Depth of Water
}

\author{
Hew Wei Ren ${ }^{1}$, Fatimah Al Zahrah Mohd Saat ${ }^{1,2,3^{*}}$, Fadhilah Shikh Anuar ${ }^{2,4}$, Mohd Arizam Abdul \\ Wahap $^{2,4}$, Ernie Mat Tokit ${ }^{1,2}$, Tee Boon Tuan ${ }^{1,2}$

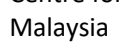

\section{Article history:}

Received 24 December 2020

Received in revised form 15 February 2021

Accepted 23 February 2021

Available online 28 February 2021

Keywords:

Hydrokinetic turbine; wake recovery;

dynamic mesh method

\begin{abstract}
Depletion of fossil fuel caused mankind to look for sustainable and green energy resources. The characteristic of hydrokinetic turbine with ability to operate at low head stream and at low cost made it a good choice for use to harness hydro source of energy. As hydrokinetic turbine gain attention from the industry player, many experimental and Computational Fluid Dynamics (CFD) studies related to hydrokinetic turbine have been carried out. Yet the relationship of flow depth variation and wake recovery behind the turbine is still not fully understood. There is limited study about the effects of flow depth variations on the wake recovery behind the turbine. In this paper, a CFD model investigation was done based on published experimental work. A hydrokinetic water turbine was drawn using the MHKF1-180 and NACA4418 foils dimensions. The transient CFD study was conducted using SST $k$ - $\omega$ turbulence model and dynamic mesh method. The results showed that in near wake region, the wake at deeper depth will recover faster seemingly due to pressure change at that depth and the faster rate of momentum transfer of the fluid. It can be concluded that the deeper the placement of the turbine inside the water channel, the faster the wake recovers. The wake recovery results as presented in this paper should be considered when placing set of turbines especially in array arrangement to obtain a more efficient energy conversion.
\end{abstract}

\section{Introduction}

Energy is one of the most important elements in the world, and a wise use of energy helps the mankind to conduct daily activities efficiently. Principle of conservation of energy states that energy cannot be created nor can it be destroyed. It can only be converted from one form to another [1]. For an example, electricity which is generated from gas power plant is a result of conversion of energy

\footnotetext{
* Corresponding author.

E-mail address: fatimah@utem.edu.my (Fatimah Al Zahrah Mohd Saat)
} 
from chemical energy (natural gas) into heat energy (mixture of superheated gas) and then into mechanical energy (rotation of gas turbine) and finally into electric energy (by generator). Energy is not only important for mankind to carry out their daily activities, but it can also bring impact to the economy. In 1970s, the economy of certain regions of the world had hard hit due to energy crisis [2]. From 19 century until today, fossil fuel is the major source of energy for many technologies. This is due to the price of fossil fuel that was cheaper at that time and the technology or method to harvest the energy is more stable compared to other sources of energy like solar energy, wind energy, hydro energy, and nuclear energy. Intemperance of mankind in using fossil fuel from 19 century until today has led to depletion of the source. Besides that, most technology comes with weak ability to efficiently convert fossil fuel into useful energy. As a result, the use of fossil fuel for many technologies led to many environmental issues like global warming and air pollution. In order to avoid these negative impacts of using fossil fuel, mankind need to find renewable energy sources or use green energy technology for the sake of securing the future of the next generation. There are many types of sources of green energy that are available in abundance like solar energy, wind energy, biomass energy, hydro energy and geothermal energy. Ehrlich and Geller [3] mentioned that among sources of renewable energy that can be used to generate electricity, the hydropower is considered one of the cheaper and a more efficient method. Hydro energy can be extracted using two methods. The first method is to extract energy from water that flows with high potential energy, for an example the water flow from a dam. Hydro energy can also be extracted using the second method where kinetic energy from flowing water like tidal wave or river stream can be converted into electricity.

The method of extraction of hydro energy from the kinetic energy of flowing water is also known as hydrokinetic energy conversion. Hydrokinetic turbine is used to extract hydrokinetic energy. According to Muratoglu and Yuce [4] the hydrokinetic turbine is an electromechanical device that is able to extract kinetic energy from the water stream into electrical energy without impoundment structure. Kumar and Sarkar [5] stated that there are several categories of hydrokinetic turbine. Examples of hydrokinetic turbine are the vertical axis turbine, horizontal axis turbine and crossflow turbine, just to name a few. Two important factors that need to be considered in the selection of hydrokinetic turbine are the characteristics of water flow (like pressure head and flow velocity) and the geographic of the location. According to Anyi and Kirke [6], the performance of axial turbine is usually better than the other turbine in the aspect of speed operation, torque fluctuation and efficiency. Due to this reason, horizontal axis turbine (one type of the axial turbines) has been selected to be the focus of the study to be reported in this paper. There are many similarities between hydrokinetic turbine and wind turbine. Published studies [7,8] stated that one of the most obvious difference between hydrokinetic turbine and wind turbine is that the hydrokinetic turbine is able to extract more energy from fluid as the density of water is higher (i.e. 800 times greater) compared to air. Based on Duvoy and Toniolo study [7], theoretical comparison between water and wind turbines showed that hydrokinetic turbine was able to generate 800 times more energy compared to wind turbine.

Researchers $[9,10]$ defined that wake of turbine is the rotationality of flow within the region downstream of the turbine which experiences disturbed flow that is characterized by the reduced flow speed, the pressure differential, expanding area, increase of turbulence and swirl. One of the terms commonly used to describe behaviors of wake is the wake recover. Jump et al., [9] defined that wake recover is a formation of shear boundary layer between the low energy wake region and surrounding ambient flow that caused the momentum from surrounding flow field to be transferred back into the wake region. The wake is dissipated through this re-energization process and the flow field recovers the loss of velocity. Many studies [9,11-13] related to wake recovery have been reported due to the significant impact of it on the performance of turbine. Wake recovery is the focus 
of researchers as wake recovery will affect the placement of turbine in array, especially the placement of hydrokinetic turbine. Unfortunately, there are very limited study related to the effect of flow depth to the wake recovery of the turbine. An example of wake recovery investigation that is related to flow depth was reported by an experimental investigation of Aghsaee and Markfort [13]. The study provided good insight on the impact of wake recovery on the placement of turbine in the water at different depths. To the authors knowledge, there is still no other publication found related to CFD studies on the effect of depth variation on the wake recovery of flow across turbines. So, there is a need to study the effects of flow depth variations on the wake recovery behind a turbine using CFD method. It is often reported that CFD results supplements the experimental findings by showing results that is difficult to be measured and shown experimentally. The CFD models of the current investigation is validated with the experimental results of Aghsaee and Markfort [13] and extension of analysis are presented in ways that were not shown by the experimental results. With the result of this study, future decision can be made to increase the performance of turbine array through the manipulation of depth of water flow. The faster the wake recovers, the more turbine can be placed in an array and therefore a much more economical benefits can be gained when generating electricity by using this hydrokinetic method.

\section{Methodology}

In this study, a CFD model was solved for the experimental work reported by Aghsaee and Markfort [13]. The computational model was solved using ANSYS FLUENT software, and the results were validated with the experimental results of Aghsaee and Markfort [13]. The results from validated models were discussed further to cover the parts that could not be shown experimentally. Figure 1 shows the side view of the model at $x$ depth. The benchmark case for this study is a model with turbine placed at a depth of $125 \mathrm{~mm}$ from the surface, following the case reported by Aghsae and Markfort [13]. Additional models were also solved where depths of turbine's placement were varied by $15 \mathrm{~mm}$ difference from the benchmark. Cases with turbine being placed at a shallower depth of $110 \mathrm{~mm}$ and a deeper depth of $140 \mathrm{~mm}$ from the surface of the water were solved. This is done to investigate the effect of flow depth variations on the wake recovery behind the turbine.

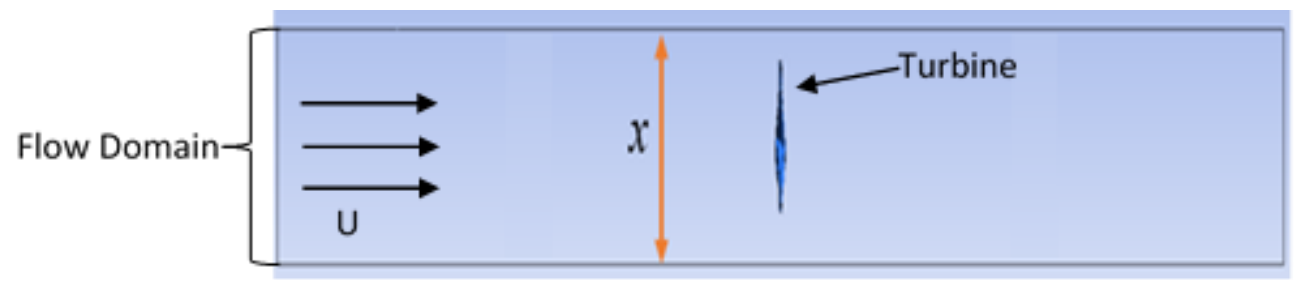

Fig. 1. Side view of model at $x$ depth. $U$ denotes velocity of fluid

A turbine with similar geometry and dimension to the one reported in Aghsaee and Markfort [13] was modelled. The geometry was obtained in the form of coordinates reported in the paper. The coordinate involves the chord length and twists angle of the turbine blade from the center of the hub. While the aerofoil type blade was drawn following the dimension of MHKF1-180, which was obtained from Shiu et al., [14]. As shown in Figure 2(a), the resulting turbine with the aerofoil blade was shown. The turbine was placed inside a square computational domain that represents the duct. The square duct (fluid domain) dimension is also similar to the duct used in the experimental study, as reported in Aghsaee and Markfort [13]. The dimension of the fluid domain of the duct was 30000 $\mathrm{mm}$ (length) $\times 900 \mathrm{~mm}$ (width) $\times 125 \mathrm{~mm}$ (height). The duct is an open channel with one surface open to the surrounding. The turbine was located at an axial location of $y=26000 \mathrm{~mm}$ from the pipe 
entrance. In the model, the fluid flows in the $y$-direction. Hence, the $y$-direction is the axial direction of flow. The location of the turbine inside the duct domain is as illustrated in Figure 2(b) The mesh generated for the computational domain is shown in Figure 2(c).

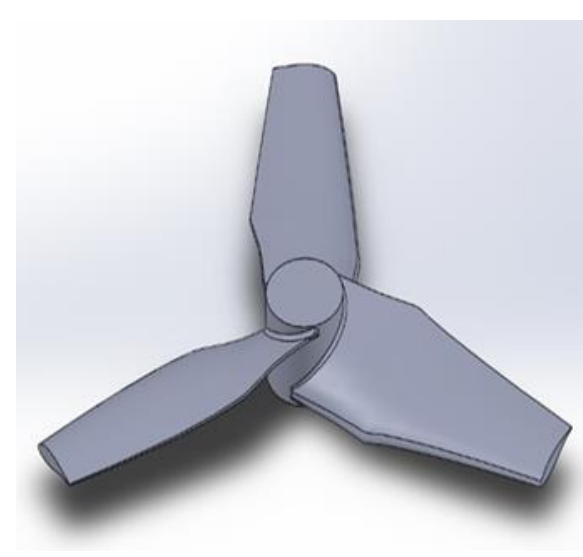

(a)

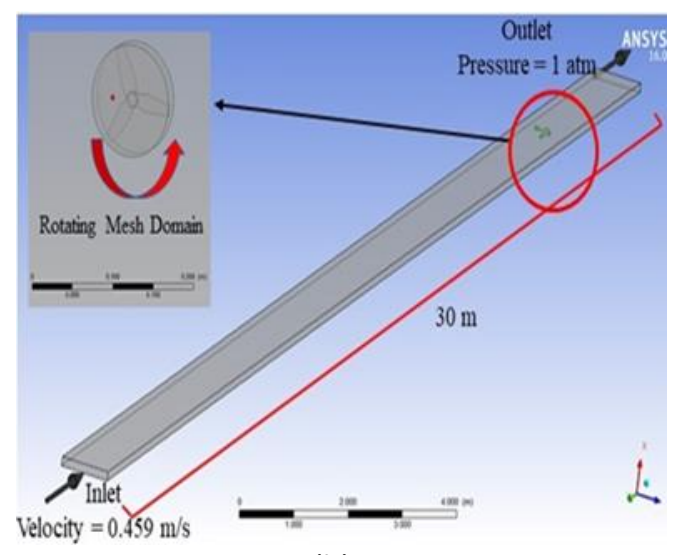

(b)

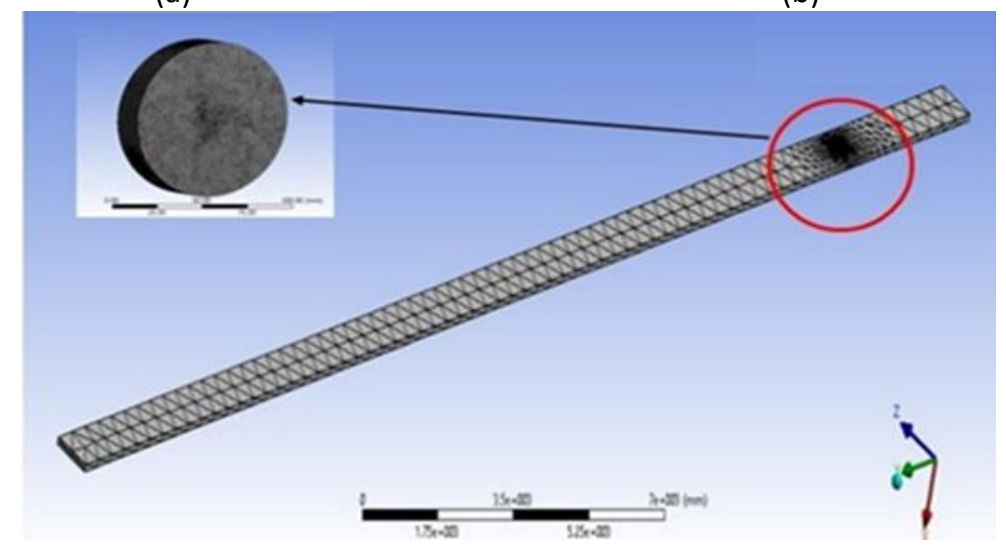

(c)

Fig. 2. (a) The hydrokinetic turbine, (b) computational domain of a $30 \mathrm{~m}$ duct with turbine placed inside the domain and (c) the mesh distribution

This CFD study was conducted using a dynamic mesh function available in ANSYS FLUENT. Mass and mass moment of inertia of turbine that were required during the setting up of the dynamic mesh were obtained from the mass properties in SOLIDWORKS. As dynamic mesh function is only available in the transient model, hence appropriate time step should be determined. The time step size was determined based on the turbine's time to complete one rotation. One rotation of the turbine was then divided by the number of frames that need to be captured. The time taken for one complete cycle of turbine rotation was obtained from the experimental work of Aghsaee and Markfort [13]. Table 1 shows the conditions that need to be defined when setting up the dynamic mesh model.

Turbulence model used in this CFD study was chosen from two equation model (a category of Reynolds Averaged Naveir Stokes (RANS) turbulence model). Two equation model was used due to its ability to provide CFD results with compromise between accuracy, complexity and robustness. Previous CFD studies [15,16] also used turbulence model from two equation model. Silva et al., [15] and Kolekar et al., [16] used Shear Stress Transport (SST) k- $\omega$ turbulence model. The SST k- $\omega$ model was reported to be performing well in many flow conditions that involved both the viscid and inviscid flow regions [17-20]. The theories or formulas for SST k- $\omega$ turbulence model is standard and can be referred to the published documentation $[16,19,20]$. 
Table 1

Setting CFD model of turbine using dynamic mesh method

\begin{tabular}{llll}
\hline \multicolumn{2}{l}{ Dynamic Mesh Zone Setting } & & \\
\hline Zone Name & Type & Six DOF UDF/Properties & Six DOF Option \\
Inner domain & Rigid body & rotate & On \& Passive \\
Turbine & Rigid body & rotate & On \\
Outer domain & Stationary & - & - \\
\hline \multicolumn{2}{l}{ Time Step Setting } & & \\
\hline Parameter & Value & \\
Time step size & 0.04 & \\
Max number of iterations & 50 & \\
Number of time step & 1000 & \\
\hline
\end{tabular}

Second order UPWIND differencing scheme was used for discretization of equations in CFD models, following the suggestion as stated by Ramayee and Supradeepan [21]. Convergence criteria in this study was determined from the Root Mean Square (RMS) residual type. The normal target for RMS residual, especially for steady model, is around $1 \times 10^{-6}$ for CFD analysis [5]. However, reaching low residual for transiet model with complicated feature of dynamic mesh is usually not feasible. In this study, the RMS residual of continuity equation was observed to be at $1 \times 10^{-3}$, while the RMS values for momentum and turbulence equations were at $1 \times 10^{-4}$. So, the transient numerical iteration for each timestep of these CFD models will be iterated until the RMS residual reached these values. Similar range of convergence was also reported especially when the model is transient in nature $[17,19]$. In this study, the average velocity of the solution was also monitored in addition to the monitoring practice of the residual convergence. It was found that the velocity reached a constant value which indicated that the solution is converged. This is as shown in Figure 3.

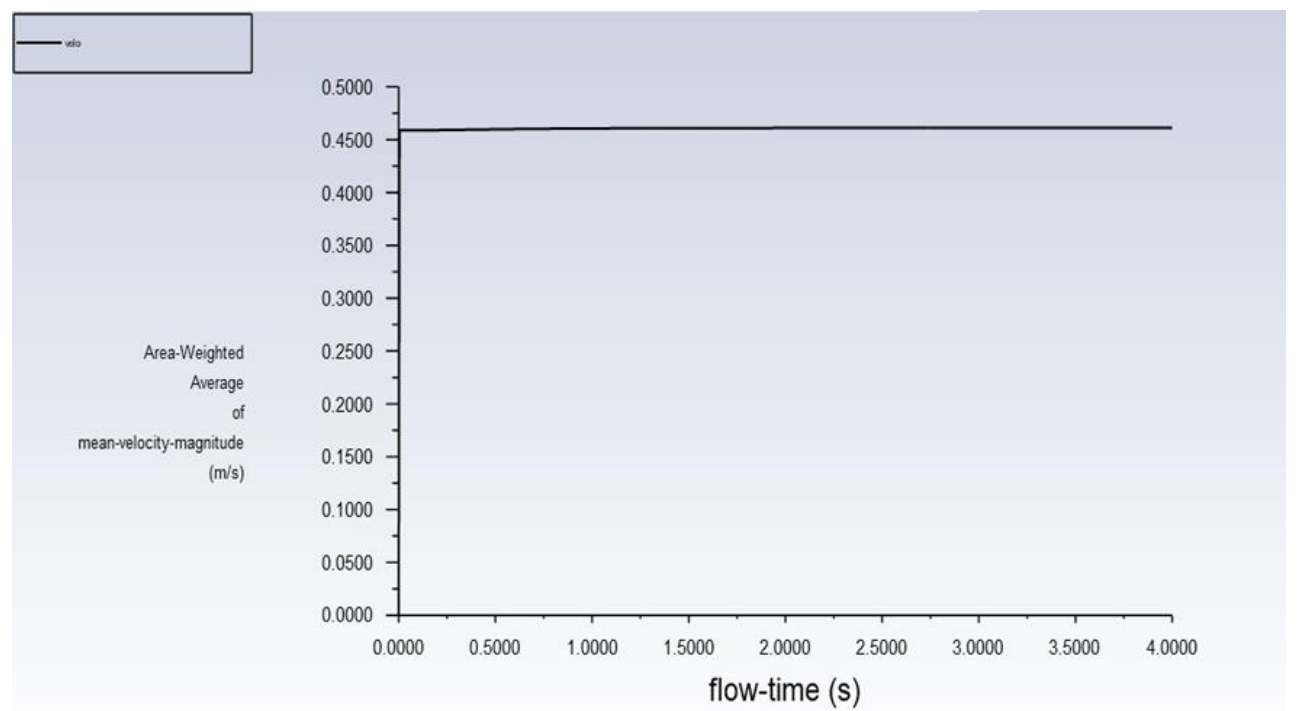

Fig. 3. Graph of mean velocity magnitude vs flow time

The fluid inside the duct was set as water, the properties of water is shown in Table 2 . The inlet boundary of the duct was set as constant velocity of $0.459 \mathrm{~m} / \mathrm{s}$ while the outlet boundary was set with atmospheric pressure. These values are set to match the benchmark experimental work used for this study. 
Table 2

Properties of water

\begin{tabular}{ll}
\hline Properties & Value \\
\hline Pressure & $1 \mathrm{~atm}$ \\
Temperature & $25^{\circ} \mathrm{C}$ \\
Density & $998.2 \mathrm{~kg} / \mathrm{m}^{3}$ \\
Viscosity & $0.001003 \mathrm{~kg} / \mathrm{ms}$ \\
Thermal Conductivity & $0.6 \mathrm{~W} / \mathrm{mK}$ \\
Specific Heat & $4182 \mathrm{~J} / \mathrm{kgK}$ \\
Turbulence Intensity & $5 \%$ \\
\hline
\end{tabular}

A grid independence test was carried out to determine the minimum number of grids required to achieve grid independence. This step is crucial as it allows the CFD study to be carried out using the least computational resources yet with accurate result. Table 3 shows the details of the grid and the resulting velocity values. The grids were refined within the dynamic mesh domain to capture the fast data changes within that region as the fluid dynamics of flow were expected to be severely changing within that region due to the turbine's rotational movement. The models were tested based on the mean stream-wise velocity; one of the crucial parameters that need to be measured in this study. Differences of approximately $2.5 \%$ in the value of velocity was detected between models with fine and medium mesh. A fine mesh was therefore used for the models.

\section{Table 3}

Details of grid and result of CFD

\begin{tabular}{lllll}
\hline Grid (level) & Skewness & No of Elements & No of Nodes & Mean Stream-Wise Velocity (m/s) \\
\hline Course (1) & 0.2408 & $1.1 \times 10^{6}$ & $2.2 \times 10^{5}$ & 0.432 \\
Medium (2) & 0.2413 & $2.7 \times 10^{6}$ & $5.4 \times 10^{5}$ & 0.450 \\
Fine (3) & 0.2439 & $1.2 \times 10^{7}$ & $2.4 \times 10^{6}$ & 0.461 \\
\hline
\end{tabular}

Investigations were done for several cases. Table 4 shows a summary of the cases conducted in this study. Two types of studies were carried out: sensitivity study and parametric study. Cases A and $B$ represent the computational domain's sensitivity study concerning the inner domain's size. The computational domain involved inner and outer domains. The inner domain was the domain that included the turbine, and the mesh within this domain was changing as the turbine rotates. The outer domain involved the domain with static mesh. The small size of the inner domain is usually used due to its attractive feature of low computational effort. However, the small size of the inner domain may lead to a solution being interrupted at the interface between the two domains. Hence care should be exercised in choosing the size of the dynamic mesh domain (inner domain) so that a good balance can be achieved between the accuracy of the solution and the computational effort.

Table 4

Summary of cases

\begin{tabular}{llll}
\hline Case & Water Depth, $x(\mathrm{~mm})$ & Inner Domain Size & Type of study \\
\hline A & 125 & small & sensitivity study \\
B & 125 & large & \\
C & 110 & large & parametric study \\
D & 140 & large & \\
\hline
\end{tabular}

The sensitivity study was done to test the sensitivity of results due to the definition of the size of the computational domain. A model that was less sensitive to computational domain size would be 
used for further investigation of parameters that was denoted as parametric study which will be represented by Cases $B, C$, and $D$.

\section{Results}

\subsection{Model Validation}

CFD models' results were compared to the results of the benchmark experimental work [13]. The results showed errors that were less than $1 \%$. This indicates that the model provided results that are almost similar to the experiment. Hence, it can be concluded that the CFD model was validated. The experimental data, CFD results and the calculated error values between the experiment and CFD are shown in Table 5.

Table 5

Comparison of CFD results with experimental results of Shiu et al., [14]

\begin{tabular}{llll}
\hline Parameter & Experiment [13] & CFD & Error (\%) \\
\hline$U_{\text {hub }}(\mathrm{m} / \mathrm{s})$ & 0.459 & 0.461 & 0.44 \\
Thrust $(\mathrm{N})$ & 0.689 & 0.695 & 0.87 \\
\hline
\end{tabular}

The term $U_{\text {hub }}$ represents the mean stream-wise velocity of the incoming flow at hub height level. The $U_{\text {hub }}$ from CFD model was taken at point $(0,0.05,0)$ which was the same location where $U_{\text {hub }}$ were reportedly taken in the experimental work [13]. Thrust was also calculated by incorporating the CFD results into Eq. (1)

$T=C_{t} 0.5 \rho A U_{h u b}^{2}$

where $T$ represents thrust, $C_{t}$ represents thrust coefficient, $\rho$ represents density, $A$ represents rotor area and $U_{\text {hub }}$ represents mean stream-wise velocity at hub height level. In the study the value of $\rho$ is $998.2 \mathrm{~kg} / \mathrm{m}^{3}, A$ is $7.85 \times 10^{-3} \mathrm{~m}^{2}$ and $C_{t}$ is 0.835 (based on blade profile characteristics).

\subsection{Results of Sensitivity Study}

In the current CFD model, the computational domain involved two domains: the domain of dynamic mesh (inner domain) and the domain with static mesh (outer domain). Selecting the size of the dynamic mesh domain can be difficult, especially for transient models. Transient models involved unsteady simulation where models were solved to capture the time history development of flow as the turbine rotates. Hence, careful consideration needs to be done to ensure that the size covered by the dynamic mesh domain is sufficient for the analysis while keeping the computational effort at a reasonable level. For this reason, a sensitivity study was conducted. The sensitivity study involved two models with different inner domain (the dynamic mesh domain). The small inner domain was 20 $\mathrm{mm}$ long, and the large inner domain was $500 \mathrm{~mm}$ long. An investigation was carried out to observe any differences between the results obtained using both domains. Figure 4 shows the result that was obtained using the computational model with small and large inner domains. Figure 4 shows that the velocity contour using a small inner domain seems disconnected at the interface between the inner domain (dynamic mesh domain) and outer domain (static mesh domain). The disconnection of results indicated that the choice of the small size of the dynamic mesh domain might not be correct as it interferes with the flow field results. Disconnection of flow is not seen in the velocity contour of flow around the turbine that was solved using the large inner domain model. For the model with the small inner domain (the top contour), the interference of flow at the interface between the inner and outer 
domains seems to affect the wake development results at the downstream location of the flow. Since the current investigation is about the wake recovery downstream of the turbine, it is vital to use a computational model with a bigger inner domain, so that wake recovery calculation is not interrupted.

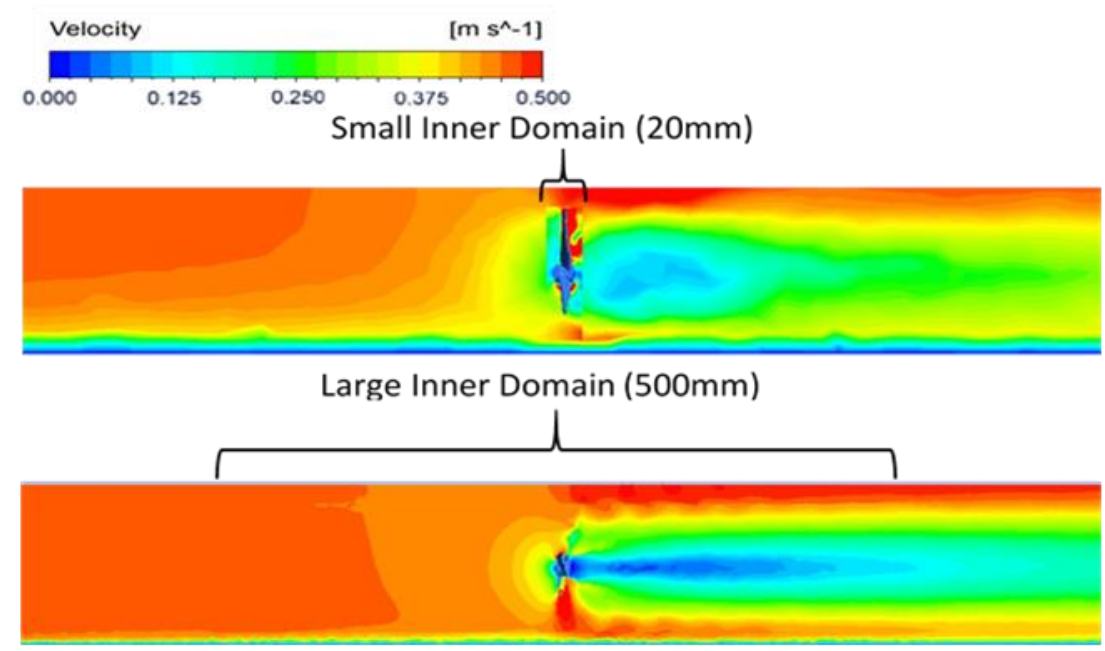

Fig. 4. Velocity contour from models with small inner domain (top) and large inner domain (bottom)

Based on this sensitivity study result, a large inner domain was chosen for the current study of the wake recovery investigations when the turbine is placed at different depth. Although the bigger inner domain provided a better result, it came with disadvantages. As the inner domain becomes larger, more computational resources were required. The increase of the inner domain size without the reduction of the size of the outer domain had increased the computational resources and time tremendously. Hence, the outer domain needs to be reduced from the original $30 \mathrm{~m}$ long to a $1 \mathrm{~m}$ long channel so that the model can be solved using a reasonable computational effort. Due to this limitation, this study can only cover the near wake investigation and not the whole domain of the far wake investigation, as reported by the experimental study of Aghsaee and Markfort [13].

\subsection{Result of Parametric Study}

Figure 5 shows the velocity contour when the turbine is placed at depths of $110 \mathrm{~mm}, 125 \mathrm{~mm}$ and $140 \mathrm{~mm}$ from the surface. The results shown in Figure 5 showed the qualitative representation of flow by using contour representation. Velocity contours are almost similar for all the cases. A clearer representation of data can be seen in Figure 6. Figure 6 shows the graph of velocity amplitude along the downstream location of the computational domain. The axial location, denoted as $y$, was defined to start from the origin (centre of the turbine) and ends at the outlet boundary at the end of the computational domain. The graph showed that the velocity of flow is highest when turbine is placed at $110 \mathrm{~mm}$ from the surface especially for downstream locations of less than $0.3 \mathrm{~m}$. This is consistent with the fact that the pressure is higher at a deeper depth, and therefore the velocity of the fluid at a deeper depth will be lower. This is also known as Bernoulli's Effect. 


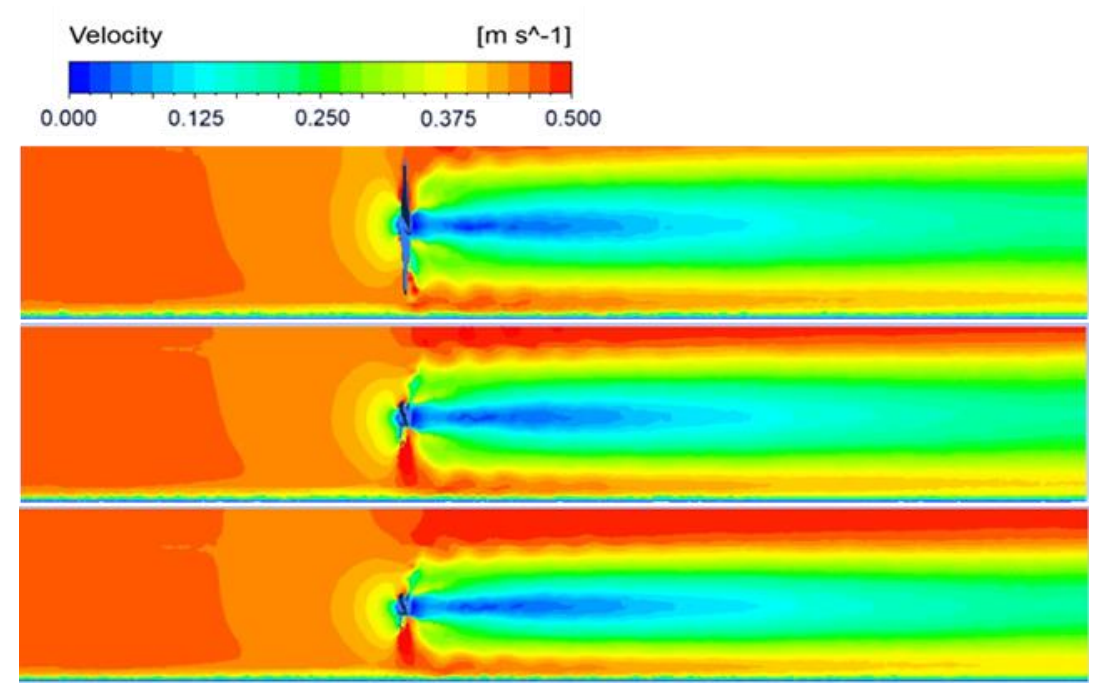

Fig. 5. Velocity contour at $110 \mathrm{~mm}$ (top), $125 \mathrm{~mm}$ (middle), $140 \mathrm{~mm}$ (bottom)

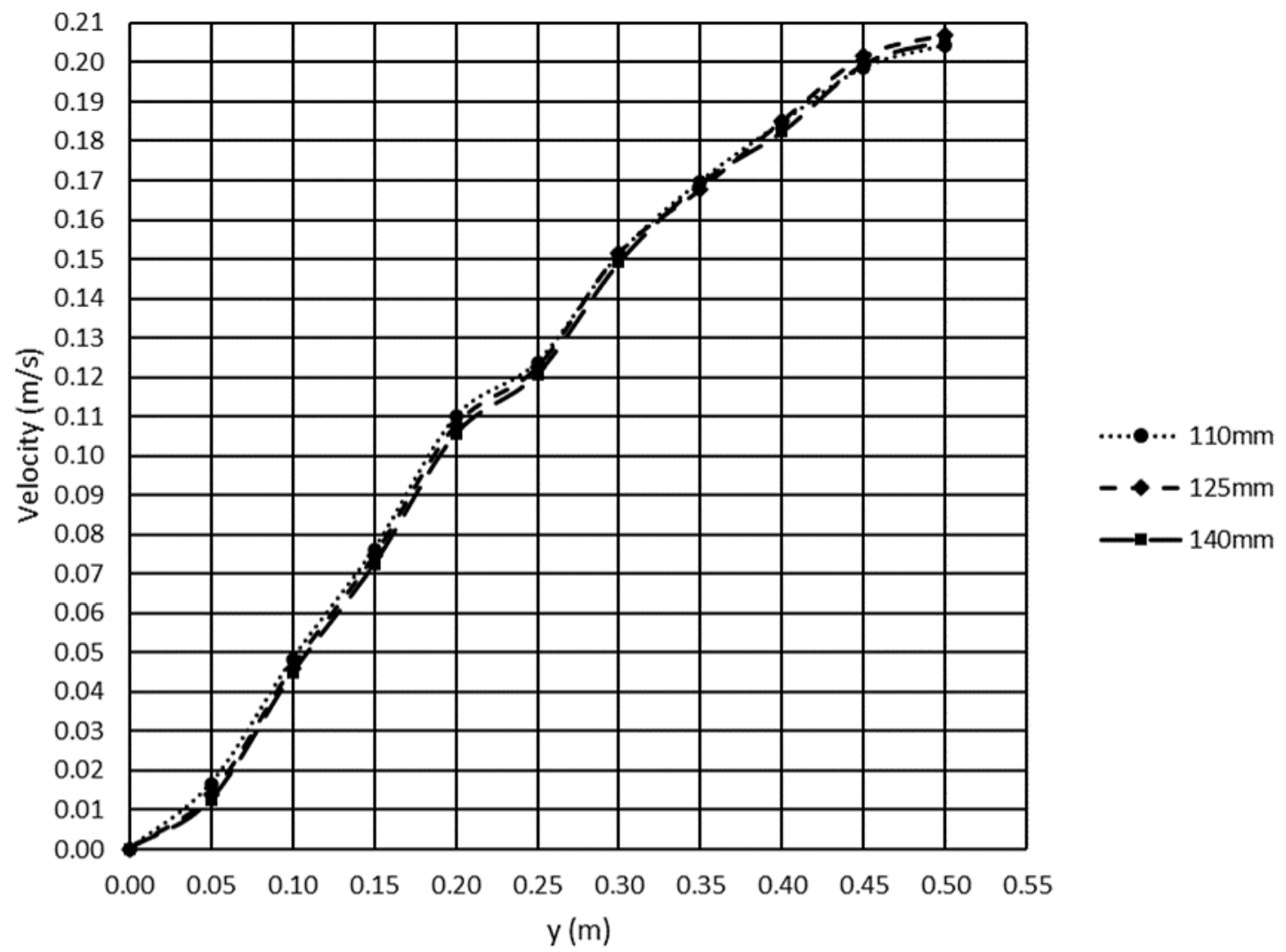

Fig. 6. Graph of Velocity vs y. y denotes distance from the origin (centre of turbine) until the boundary end of model

As the wake started to recover, after the fluid flow through the hydrokinetic turbine, the velocity of flow for cases with turbine installed at deeper depth became larger than that of the $110 \mathrm{~mm}$ case. This can be seen for downstream locations exceeding the location of $0.3 \mathrm{~m}$ from the turbine. At these locations, the velocity amplitude of the flow with turbine placed at $125 \mathrm{~mm}$ is highest compared to other cases. At locations after $y=0.45 \mathrm{~m}$, the velocity of flow for turbine that is placed at a deeper depth of $140 \mathrm{~mm}$ is also seen to be higher than that of the $110 \mathrm{~mm}$ case. This indicates that wake recovers faster when turbine is placed deep into the water. The recovery of wake leads to higher 
velocity of the flow at downstream locations. Although the difference of velocity between cases with turbine placed at different depths is not significant (due to the limitation of model which is able to show only the velocity of near wake region), the result is still in good agreement with the results obtained by Aghsaee and Markfort [13]. The results showed that the deeper the depth of the turbine placement into the water, the faster the wake recover.

The wake recovery phenomena can also be assessed via vortex plots, as shown in Figure 7. It can be seen that the vortex pattern is not symmetrical with respect to the centre of the turbine. The vortex sheds to a larger distance on top and a shorter distance at the bottom. This asymmetrical feature shows that the wake is bigger at locations nearer to the surface. This is logical since pressure increases as the depth into the water increases. Hence, wake could recover faster at location deeper in the water. Comparison of vortex plots for all cases of turbine placements $(110 \mathrm{~mm}, 125 \mathrm{~mm}$ and $140 \mathrm{~mm}$ ) shows that vortex reduces its strength faster as the turbine is placed deeper into the water. This again proves that the wake recovers faster at a deeper depth.

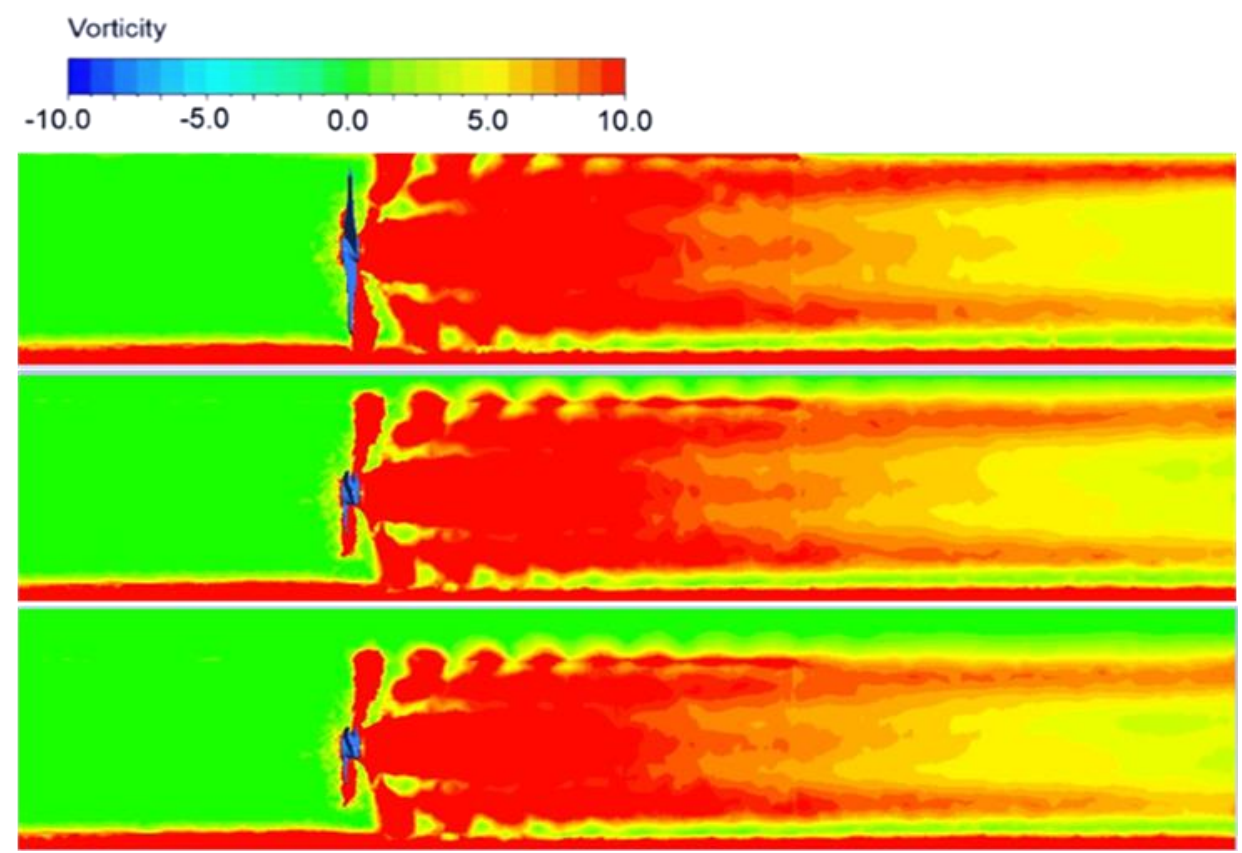

Fig. 7. Vorticity Contour at $110 \mathrm{~mm}$ (top), $125 \mathrm{~mm}$ (middle), $140 \mathrm{~mm}$ (bottom)

The wake recovery phenomena were supported further with results of a streamline plot. Figure 8 shows the streamline of flow when the turbine is placed at a different depth, starting from $110 \mathrm{~mm}$ depth as shown on top of Figure 8 , followed by $125 \mathrm{~mm}$ depth and $140 \mathrm{~mm}$ depth. As shown in Figure 8 , when the depth increases from $110 \mathrm{~mm}$ to $140 \mathrm{~mm}$, the intensity of flow at locations near the surface (the streamlines on top of the plots), especially at the downstream location, increases. As the turbine is placed deeper into the water, there will be more space for fluid to flow on top of the turbine and near the surface. The turbine's presence will create turbulence that helps the fluid at locations near the surface to flow downstream. The quantity of surrounding ambient flow field will affect the rate of wake recovery. According to Jump et al., [9] and Gómez-Elvira et al., [22], wake recovery is the re- energisation process where the flow field recovers the lost velocity by transfer of momentum. Welty et al., [23] stated that the momentum would transfer from the faster moving layer to the slower moving layer. In other words, the momentum will transfer from the surrounding ambient flow field to the low energy wake region. The low energy wake region is shown as the disturbed streamline at the turbine's central location. The strength of flow is represented by a weak streamline (light blue 
lines). A more stabilised streamline represents the surrounding fluid with strong amplitude (red lines). So, when the influence of the surrounding fluid is stronger, the rate of wake recovery seems faster. This can be seen clearly by comparing the flow downstream and the flow at the turbine's central location. The disturbed streamline (the wake) becomes shorter as the turbine is placed deeper into the water. To make this explanation easier to be understood, the streamline can be treated as a wire. The larger the quantity of wire, the more electricity can be transferred. So does the streamline or surrounding flow field. The larger the surrounding flow field, the more momentum it can transfer to recover the lost velocity and the faster the wake recovers.

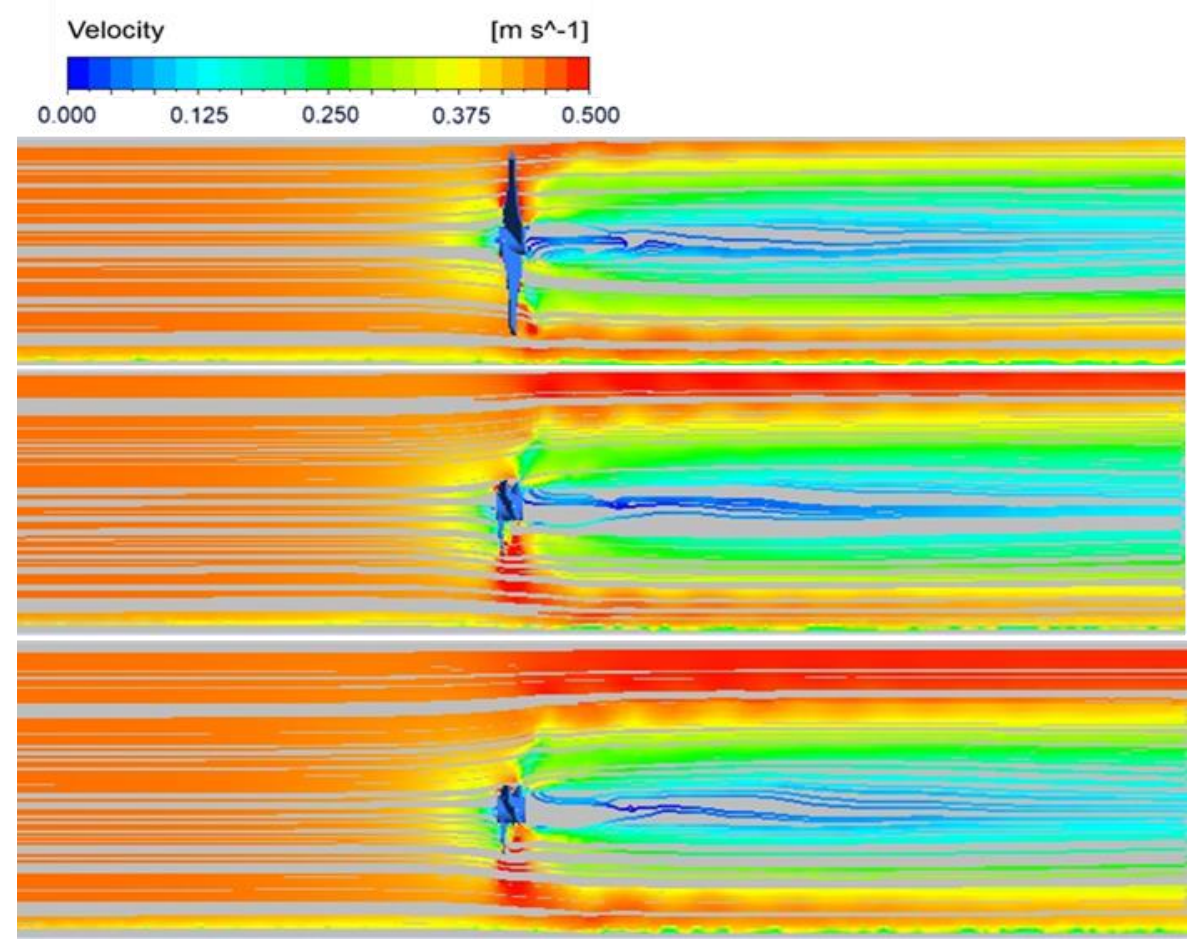

Fig. 8. Streamline at $110 \mathrm{~mm}$ (top), $125 \mathrm{~mm}$ (middle), $140 \mathrm{~mm}$ (bottom)

As shown in Figure 8, when the turbine was placed at the deepest location (140 $\mathrm{mm}$ depth), the surrounding ambient flow field is the largest and the strength of velocity of the mean flow is bigger. More momentum can be transferred to the wake to recover the lost velocity. As the depth of turbine placement reduces, the surrounding ambient flow field also reduces. This will lower the momentum transfer rate, thus slowing wake recovering process.

The model is solved for a longer period to look at the change of flow with respect to time. This helped in identifying the stage of the flow in the solved models. Figure 9 shows the development of vortex with time for two cases: the $110 \mathrm{~mm}$ depth and the $125 \mathrm{~mm}$ depth of turbine placements. As shown in Figure 9, the vortex for both $110 \mathrm{~mm}$ depth and $125 \mathrm{~mm}$ depth are almost similar as time increases. This indicates that the solution had reached a steady state situation. In steady state situation, the fluid dynamics of flow did not change with time. Therefore, similar behaviour is expected throughout the process as the turbine rotates. Similar behaviour was also seen for the case of $140 \mathrm{~mm}$ depth. The result in $140 \mathrm{~mm}$ depth is not shown in this paper to avoid unnecessary repetitions. 


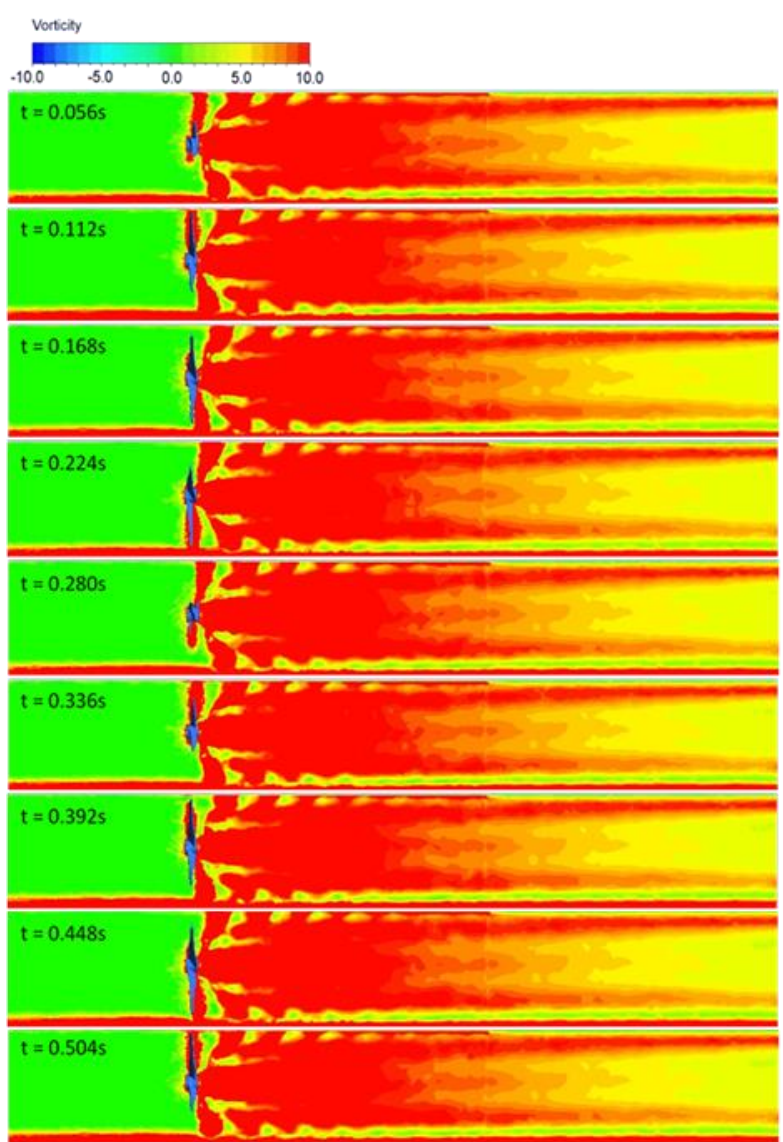

(a)

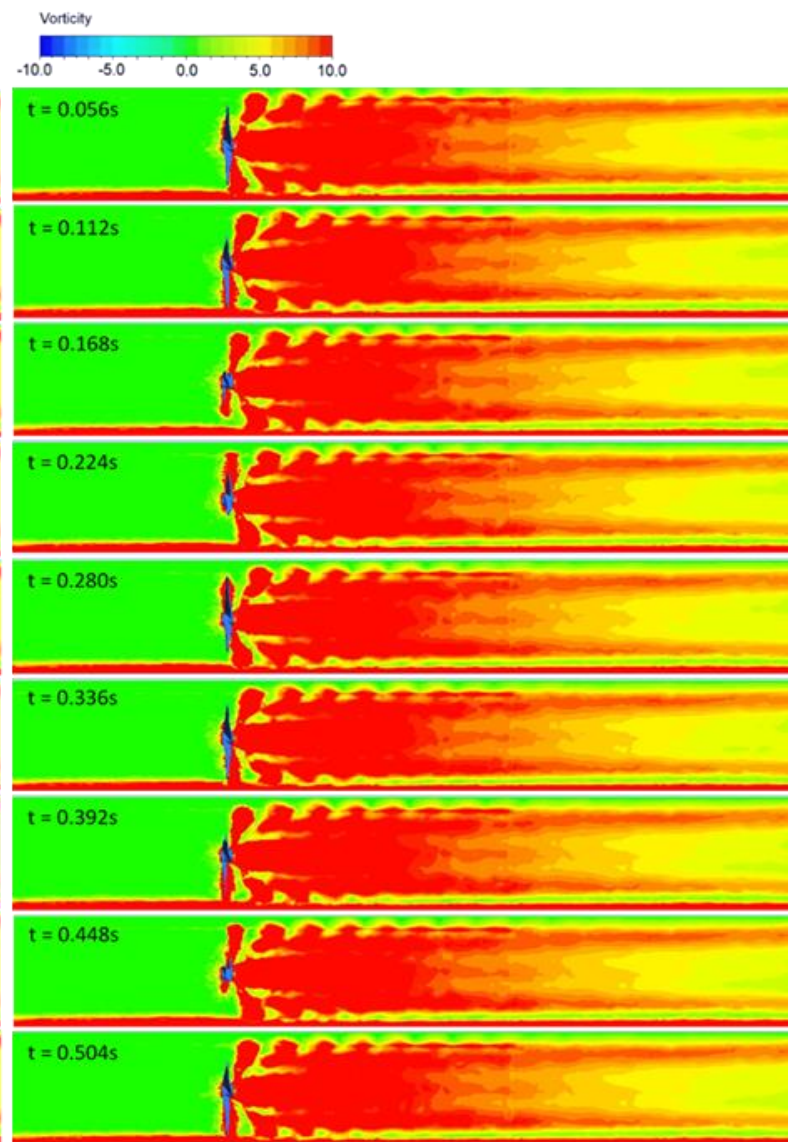

(b)

Fig. 9. Development of vorticity contour with time for cases of turbine placed at depths of (a) 110 $\mathrm{mm}$ and (b) $125 \mathrm{~mm}$

The power at different depths was calculated by incorporating the CFD results into Eq. (2) to determine whether the effect of flow depth variation will affect a single turbine's performance. Table 6 shows the turbine's thrust values when the turbine is placed at different depths inside the water. The power, $P$, was calculated as

$$
P=C_{p} 0.5 \rho A U_{h u b}^{3}
$$

where $P$ represents power, $C_{p}$ represents power coefficient, $\rho$ represents density, $A$ represents rotor area and $U_{\text {hub }}$ represents mean stream-wise velocity at hub height level.

Table 6

Comparison of power of turbine at difference depths

\begin{tabular}{lll}
\hline Depth $(\mathrm{mm})$ & $U_{\text {hub }}(\mathrm{m} / \mathrm{s})$ & Power $(\mathrm{W})$ \\
\hline 110 & 0.461 & 0.228 \\
125 & 0.461 & 0.228 \\
140 & 0.461 & 0.228 \\
\hline
\end{tabular}

In this study $\rho$ is $998.2 \mathrm{~kg} / \mathrm{m}^{3}, A$ is $7.85 \times 10^{-3} \mathrm{~m}^{2}$ and $C_{p}$ is 0.593 (based on Betz Law). According to Yuce and Muratoglu [4], Betz Law proposed that for a free-flow horizontal axis turbine, the maximum theoretical power coefficient, $C_{p}$, is 0.593 . In other word, the maximum power that is able to be produce by a free-flow horizontal axis turbine is $59.3 \%$. Ehrlich and Geller [3] mentioned that 
the common power coefficient of free-flow horizontal axis turbine is in the range of $35 \%$ to $45 \%$. The $C_{p}$ in the current calculation is set constant based on the Betz Limit with assumption that the turbine is able to produce maximum power according to this limit.

From the results shown in Table 6, it can be observed that the depth that was investigated in this study does not give a significant impact on the power of a single turbine when placed inside the water. This is probably due to the small differences of depth between one case to another and the inlet velocity was treated the same for all the cases. As a result, the $U_{\text {hub }}$ is almost constant for all cases. However, a more meaningful understanding was obtained form visualisation of flow as reported in Figure 6. Figure 6 showed that as the depth of water increases, the faster the wake recovers. Once turbines are placed in an array, the power generated by the turbine arrays might be impacted due to the wake that develops at the back of each turbine. Hence increasing the depth of turbine placement into the water would increase the performance of arrays of turbine because the wake recovers faster. This will enable more turbine to be put in the array, and therefore more electricity can be generated by using this hydrokinetic method. More significant results in wake recovery were expected to be observed for extended investigation covering the study from near wake to the far wake regions. However, a suitable method will need to be developed to minimize the computational efforts needed to solve the models.

\section{Conclusions}

The computational fluid dynamics investigations reported in this paper agrees with the experimental results of Aghsaee and Markfort [13]. As reported in the current study, computational models provided additional information related to flow visualization within the area of flow that is affected by the presence of the turbine. This helped in the understanding of the fluid dynamics of flow related to the wake recovery phenomenon of flow when turbine is used to extract energy from the water. It is clear that the deeper the depth of turbine's placement, the faster the wake recovers. This is because the larger surrounding flow field will enable a faster momentum transfer rate when the turbine is placed deeper into the water. The finding is essential in helping the engineer to design a more efficient hydrokinetic turbine array. Sensitivity studies also show that it is better to use a large inner domain (dynamic mesh domain) as it shows more accurate and reliable results than the model with a small inner domain (dynamic mesh domain). Future investigations that cover a larger domain should be considered to help understand far wake as it could give a clearer picture of flow depth variations on the wake recovery behind the turbine. This, of course, will require the use of a highperformance computer. With all the reported limitations, this study provides an insight into the importance of the proper selection of computational domain and modelling, especially in the field of a hydrokinetic turbine.

\section{Acknowledgement}

This research work was conducted using facilities at Universiti Teknikal Malaysia Melaka. The authors also acknowledge allocation under JURNAL/2019/FKM/Q00018.

\section{References}

[1] Haseli, Yousef. Entropy Analysis in Thermal Engineering Systems. Academic Press, 2019.

[2] Coyle, Eugene D., and Richard A. Simmons. Understanding the global energy crisis. Purdue University Press, 2014. https://doi.org/10.26530/OAPEN 469619

[3] Ehrlich, Robert, and Harold A. Geller. Renewable energy: a first course. CRC press, 2017.

[4] Muratoglu, Abdullah, and M. Ishak Yuce. "Design of a river hydrokinetic turbine using optimization and CFD simulations." Journal of Energy Engineering 143, no. 4 (2017): 04017009. 
https://doi.org/10.1061/(ASCE)EY.1943-7897.0000438

[5] Kumar, Dinesh, and Shibayan Sarkar. "A review on the technology, performance, design optimization, reliability, techno-economics and environmental impacts of hydrokinetic energy conversion systems." Renewable and Sustainable Energy Reviews 58 (2016): 796-813. https://doi.org/10.1016/i.rser.2015.12.247

[6] Anyi, Martin, and Brian Kirke. "Evaluation of small axial flow hydrokinetic turbines for remote communities." Energy for Sustainable Development 14, no. 2 (2010): 110-116.

https://doi.org/10.1016/i.esd.2010.02.003

[7] Duvoy, Paul, and Horacio Toniolo. "HYDROKAL: A module for in-stream hydrokinetic resource assessment." Computers \& geosciences 39 (2012): 171-181.

https://doi.org/10.1016/i.cageo.2011.06.016

[8] Salleh, Mohd Badrul, Noorfazreena M. Kamaruddin, and Zulfaa Mohamed-Kassim. "Savonius hydrokinetic turbines for a sustainable river-based energy extraction: A review of the technology and potential applications in Malaysia." Sustainable Energy Technologies and Assessments 36 (2019): 100554.

https://doi.org/10.1016/i.seta.2019.100554

[9] Jump, Ellen, Alasdair Macleod, and Tom Wills. "Review of tidal turbine wake modelling methods." International Marine Energy Journal 3, no. 2 (2020): 91-100.

https://doi.org/10.36688/imej.3.91-100

[10] McKay, Phillip, Rupp Carriveau, David SK Ting, and Timothy Newson. "Turbine Wake Dynamics." In Advances in Wind Power. IntechOpen, 2012. https://doi.org/10.5772/53968

[11] Tian, Wenlong, James H. VanZwieten, Parakram Pyakurel, and Yanjun Li. "Influences of yaw angle and turbulence intensity on the performance of a $20 \mathrm{~kW}$ in-stream hydrokinetic turbine." Energy 111 (2016): 104-116. https://doi.org/10.1016/i.energy.2016.05.012

[12] Boudreau, Matthieu, and Guy Dumas. "Comparison of the wake recovery of the axial-flow and cross-flow turbine concepts." Journal of Wind Engineering and Industrial Aerodynamics 165 (2017): 137-152. https://doi.org/10.1016/i.jweia.2017.03.010

[13] Aghsaee, Payam, and Corey D. Markfort. "Effects of flow depth variations on the wake recovery behind a horizontalaxis hydrokinetic in-stream turbine." Renewable Energy 125 (2018): 620-629.

https://doi.org/10.1016/i.renene.2018.02.137

[14] Shiu, Henry, C. P. Van Dam, Erick Johnson, Matthew Barone, Ryan Phillips, William Straka, Arnold Fontaine, and Michael Jonson. "A design of a hydrofoil family for current-driven marine-hydrokinetic turbines." In International Conference on Nuclear Engineering, vol. 44984, pp. 839-847. American Society of Mechanical Engineers, 2012. https://doi.org/10.1115/ICONE20-POWER2012-55224

[15] Silva, Paulo ASF, TAYGOARA F. OLIVEIRA, Antonio CP Brasil Junior, and Jerson RP Vaz. "Numerical study of wake characteristics in a horizontal-axis hydrokinetic turbine." Anais da Academia Brasileira de Ciências 88, no. 4 (2016): 2441-2456. https://doi.org/10.1590/0001-3765201620150652

[16] Kolekar, Nitin, Suchi Subhra Mukherji, and Arindam Banerjee. "Numerical modeling and optimization of hydrokinetic turbine." In Energy Sustainability, vol. 54686, pp. 1211-1218. 2011. https://doi.org/10.1115/ES2011-54252

[17] Allafi, Waleed Almukhtar, Fatimah Al Zahrah Mohd Saat, and Xiaoan Mao. "Fluid dynamics of oscillatory flow across parallel-plates in standing-wave thermoacoustic system with two different operation frequencies." Engineering Science and Technology, an International Journal 24, no. 1 (2021): 41-49.

https://doi.org/10.1016/i.jestch.2020.12.008

[18] Ng, Yu Han, Wah Yen Tey, Lit Ken Tan, Gerald Pacaba Arada, and M. W. Muhieldeen. "Numerical Examination on Two-Equations Turbulence Models for Flow Across NACA 0012 Airfoil with Different Angle of Attack." CFD Letters 12, no. 2 (2020): 22-45.

[19] Lin, Chou Aw, Fatimah Al-Zahrah Mohd Sa'at, Fadhilah Shikh Anuar, Mohamad Firdaus Sukri, Mohd Zaid Akop, and Zainuddin Abdul Manan. "Heat Transfer Across Tube Banks With a Passive Control Vortex Generator in Steady OneDirectional and Oscillatory Flows." CFD Letters 13, no. 1 (2021): 1-18. https://doi.org/10.37934/cfdl.13.1.118.

[20] Fluent, A. N. S. Y. S. "ANSYS fluent theory guide 15.0." ANSYS, Canonsburg, PA 33 (2013).

[21] Ramayee, L., and K. Supradeepan. "Grid convergence study on flow past a circular cylinder for beginners." In AIP Conference Proceedings, vol. 2317, no. 1, p. 030020. AIP Publishing LLC, 2021. https://doi.org/10.1063/5.0036230 
[22] Gomez-Elvira, Rafael, Antonio Crespo, Emilio Migoya, Fernando Manuel, and Julio Hernández. "Anisotropy of turbulence in wind turbine wakes." Journal of wind engineering and industrial aerodynamics 93, no. 10 (2005): 797814.

https://doi.org/10.1016/i.jweia.2005.08.001

[23] Welty, James, Gregory L. Rorrer, and David G. Foster. Fundamentals of momentum, heat, and mass transfer. John Wiley \& Sons, 2020. 